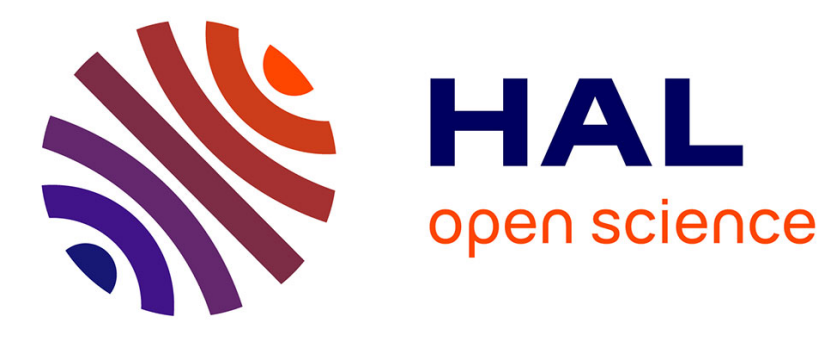

\title{
Rheology of sedimenting particle pastes
}

\author{
Abdoulaye Fall, Henri de Cagny, Daniel Bonn, Guillaume Ovarlez, Elie
}

Wandersman, Joshua Dijksman, Martin van Hecke

\section{To cite this version:}

Abdoulaye Fall, Henri de Cagny, Daniel Bonn, Guillaume Ovarlez, Elie Wandersman, et al.. Rheology of sedimenting particle pastes. Journal of Rheology, 2013, 57 (4), pp.1237-1246. 10.1122/1.4809732 . hal-00925901

\section{HAL Id: hal-00925901 \\ https://hal.science/hal-00925901}

Submitted on 9 Jan 2014

HAL is a multi-disciplinary open access archive for the deposit and dissemination of scientific research documents, whether they are published or not. The documents may come from teaching and research institutions in France or abroad, or from public or private research centers.
L'archive ouverte pluridisciplinaire HAL, est destinée au dépôt et à la diffusion de documents scientifiques de niveau recherche, publiés ou non, émanant des établissements d'enseignement et de recherche français ou étrangers, des laboratoires publics ou privés. 


\title{
Rheology of sedimenting particle pastes
}

\author{
Abdoulaye Fall, ${ }^{\text {a) }}$ Henri de Cagny, and Daniel Bonn \\ Van der Waals-Zeeman Institute, IoP, University van Amsterdam, \\ Science Park 904, 1098 XH Amsterdam, The Netherlands \\ Guillaume Ovarlez \\ Université Paris Est-Institut Navier, 2 allée Kepler, 77420 Champs-sur-Marne, \\ France
}

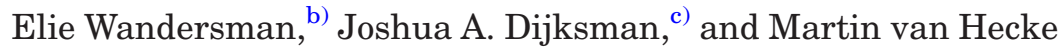 \\ Kamerlingh Onnes Laboratory, LION, Universiteit Leiden, P.O. Box 9504, \\ 2300 RA Leiden, The Netherlands
}

(Received 19 September 2012; final revision received 17 May 2013; published 26 June 2013)

\begin{abstract}
Synopsis
We study the local and global rheology of non-Brownian suspensions in a solvent that is not density-matched, leading to either creaming or sedimentation of the particles. Both local and global measurements show that the incomplete density matching leads to the appearance of a critical shear rate above which the suspension is homogenized by the flow, and below which sedimentation or creaming happens. We show that the value of the critical shear rate and its dependence on the experimental parameters are governed by a competition between the viscous and the gravitational forces, and present a simple scaling model that agrees with the experimental results from different types of experiments (local and global) in different setups and systems. (C) 2013 The Society of Rheology. [http://dx.doi.org/10.1122/1.4809732]
\end{abstract}

\section{INTRODUCTION}

Dense suspensions exhibit very rich behavior: Some suspensions exhibit a yield stress [Husband et al. (1993); Ancey and Coussot (1999); Prasad et al. (1995); Huang et al. (2005); Huang and Bonn (2007); Ovarlez et al. (2006)], many show shear banding [Barentin et al. (2004); Huang et al. (2005); Ovarlez et al. (2006); Fall et al. (2009); Schall and van Hecke (2010)], and some show shear thickening [Fall et al. (2008); Fall

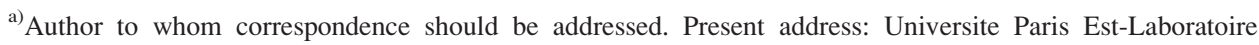
Navier, 2 allee Kepler, 77420 Champs-sur-Marne, France. Electronic mail: abdoulaye.fall@ifsttar.fr

${ }^{b)}$ Also at: Laboratoire Jean Perrin, FRE 3231, CNRS/Université Pierre et Marie Curie, Ecole Normale Supérieure, 24 rue Lhomond, 75005 Paris, France.

${ }^{c}$ Also at: Physics Department, Duke University, Science Dr, Box 90305, Durham, North Carolina 27708.
} 
et al. (2012); Brown and Jaeger (2009, 2011, 2012); Brown et al. (2010); Cheng et al. (2011)], normal stresses [Morris et al. (1999); Zarraga et al. (2000)] and shear-induced migration [Morris et al. (1999); Ovarlez et al. (2006); Fall et al. (2010)] that remain incompletely understood. A crucial role is played by the density mismatch between the particles and the fluid [Fall et al. (2009)]. Non-density-matched suspensions, such as sand in water, share features with both dry granular media and density-matched suspensions [Dijksman et al. (2010)].

On the one hand, dry granular media are collections of non-Brownian particles that interact only when in contact. Their interaction is dissipative, dominated by inelastic collisions and frictional contacts. In spite of these seemingly simple interactions, their collective behavior is very rich and complex, and has attracted much attention in recent years [Jaeger et al. (1996); GDR MiDi (2004)]. With the exception of very rapid flows [Goldhirsch (2003)], in most cases friction dominates. This then suggests that the resistance to flow, a subject of considerable fundamental interest and practical importance, is also frictional. While there remain, in particular for very slows flows, several open questions [Fenistein and van Hecke (2003); Fenistein et al. (2004, 2006)], the relation between stress and strain rate has been studied in much detail, and there is little doubt that they indeed are very similar to dry friction: The shear stress is proportional to the normal stress, only weakly varies with the strain rate, and reaches a finite yield stress in the limit of vanishing strain rate [Jop et al. (2005); GDR MiDi (2004); Dijksman et al. (2010); Dijksman et al. (2011)].

On the other hand, density-matched suspensions behave as Newtonian liquids, with an effective viscosity which grows rapidly with the packing fraction [Bonnoit et al. (2010a,b)]. It has recently been suggested [Boyer et al. (2011)] that it is possible to reinterpret this growth of the viscosity, observed at constant packing fraction, as arising via the growth of the normal stresses. Nevertheless, the rheology is very different from dry granular media - the stresses are proportional to the strain rate and thus vanish when the strain rate goes to zero. To observe this absence of a yield stress, it is crucial that the density matching is very precise-any density mismatch will lead to a finite yield stress [Fall et al. (2009)].

For the general case of non-density-matched suspensions, recent experiments point to the existence of (at least) two distinctly different flow regimes. There is a slow flow regime in which the contacts between particles are essentially frictional and the rheology is similar to that of dry granular media, while for fast enough flows, the particles are resuspended, lose contact, and the rheology becomes Newtonian [Fall et al. (2009); Møller et al. (2009); Dijksman et al. (2010)]. Prasad and Kytömaa observed a transition from a frictional to a viscous regime when shearing the sample at constant solids fraction; in contrast with measurements taken with a Couette cell, the shear stress was a decreasing function of the shear rate in the frictional regime, which led to instabilities [Prasad and Kytömaa (1995)]. The crucial question then becomes: What is the critical strain rate where the crossover between these two regimes takes place?

Here we probe the flow of a range of non-density-matched suspensions by a combination of magnetic resonance imaging (MRI), stress controlled rheometry, and rate controlled rheometry. In all cases, we observe a critical strain rate: Stress controlled rheometry evidences this critical strain rate via a viscosity bifurcation, while rate controlled rheometry (and MRI) evidences the critical strain in the rheological curves. We will show that the crossover strain rate is proportional to the density mismatch and granular pressure, thus evidencing that it can be understood to arise from a balance of static, frictional stresses to the dynamic, viscous stresses, and introduce a scaling model which is in full agreement with our data. 


\section{RHEOLOGICAL CURVES AND CRITICAL FLOW RATES}

In a first series of experiments, we probe the local and global rheology of dense suspensions composed of noncolloidal monodisperse spherical particles [polystyrene beads, diameter $40 \mu \mathrm{m}$, polydispersity $<5 \%$, density (1050) $\mathrm{kg} \mathrm{m}^{-3}$ ] immersed in a Newtonian fluid (water $+\mathrm{NaI}$ ) with a viscosity of $1 \mathrm{mPa} s$ for volume fraction $58 \% \leq \varphi \leq 63 \%$; most representative results presented here are obtained at $60 \%$. By varying the salt concentration, we can not only perfectly match the solvent and particle densities but also reach a wide range of density differences between the solvent and the particles. In all cases where we have a density difference, we keep a relatively high salt concentration, implying that the particles cream rather than sediment [Fall et al. (2009)].

MRI rheometry is performed in a wide-gap Couette geometry $\left(R_{i}=41.5 \mathrm{~mm}\right.$, $R_{o}=60 \mathrm{~mm}, H=110 \mathrm{~mm}$ ). Local velocity and concentration profiles in the flowing sample were obtained through MRI techniques described in detail in Rodts et al. (2004) and Bonn et al. (2008). For all experiments, in order to avoid slip at the walls, sandpaper of roughness equivalent to that of the particles is glued on the walls; we checked on the velocity profiles that there is no observable slip. We investigated the stationary flows for inner cylinder rotational velocity $\Omega$ ranging between $10^{-3}$ and 0.16 rps.

The MRI velocity profiles reveal an important difference between suspensions that are density matched and those that are not. The latter shows marked shear banding for the lowest $\Omega$, that is not present for the density-matched system that has a viscous behavior with nonthreshold: Gravity seems to be the motor of the shear localization. Moreover, the velocity profiles in the non-density-matched suspension show that the velocity profile falls down abruptly to 0 at the interface between the flowing and the nonflowing part: There is a critical shear rate $\dot{\gamma}_{c}$ below which no stable flow exists [Fall et al. (2009)].

Therefore, by combining the local shear stress and local strain rate, we will determine the local rheology of our suspensions. The stress is found by measuring the torque necessary to turn the inner cylinder and using the well-known $1 / r^{2}$ variation of the shear stress in a Couette geometry (which follows from momentum balance). The shear rate can be deduced from the velocity $v(r)$ profile as $\dot{\gamma}=-r \partial(v / r) / \partial r$ - so we compute for each distance $r_{i}$ the shear rate from the values of the velocity between two points

$$
\dot{\gamma}\left(r_{i}\right)=-r_{i}\left(v_{i+1} / r_{i+1}-v_{i-1} / r_{i-1)}\right) /\left(r_{i+1}-r_{i-1}\right) .
$$

In Fig. 1, we show the resulting local rheological curves for both a density matched $(\Delta \rho=0)$ and non-density-matched $\left(\Delta \rho=150 \mathrm{~kg} / \mathrm{m}^{3}\right)$ suspensions. The density-matched material flows as a Newtonian fluid (a slope of 1 on the log-log scale implies a constant viscosity) whereas a yield stress of about 3.2 $\mathrm{Pa}$ is observed for the non-density-matched system. As the figure shows, the local rheology data for the nonmatched system can be fitted by a Bingham model: $\sigma=(3.2+5.1 \dot{\gamma}) \mathrm{Pa}$ although the range of stresses is small.

In the global rheology, we probe the non-density-matched suspension $\left(\Delta \rho=150 \mathrm{~kg} / \mathrm{m}^{3}\right)$ with a vane-in-cup geometry (inner cylinder radius $R_{i}=12.5 \mathrm{~mm}$, outer cylinder radius $R_{o}=18.5 \mathrm{~mm}$, height $H=45 \mathrm{~mm}$ ) on a commercial rheometer (Bohlin C-VOR 200). Since the creaming is time-dependent, the rheology of such suspensions is time-dependent also. Applying a sufficiently high shear stress leads to a homogenization of the system, whereas for small shear stresses, the system sediments (or creams, depending on the sign of the density difference) until a layer forms that has a density very close to random close packing. It is this layer that has a yield stress because there are particle contacts: Creaming will generate a yield stress - the viscosity will then increase in time without bound, in turn implying an infinite viscosity in steady state. Both the viscosity and the yield stress are thus timedependent, and this leads to a viscosity bifurcation in the system as a function of the 


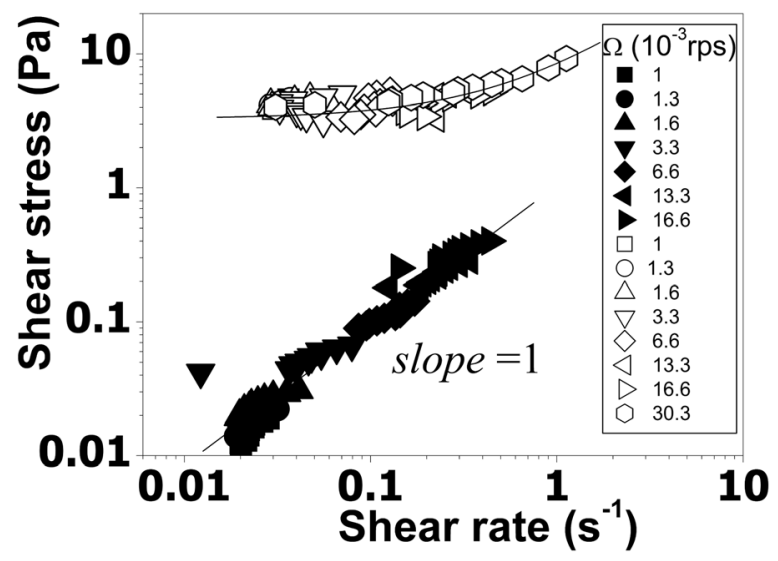

FIG. 1. Constitutive law from the MRI measurements in the density-matched (closed symbols) suspension which shows a Newtonian behavior and in the mismatched suspension (open symbols) where a yield stress behavior is observed. The continuous line is a Bingham model $\sigma=(3.2+5.1 \dot{\gamma}) \mathrm{Pa}$. The line through the Newtonian behavior gives a slope of unity. Up to the highest strain rates shown here, no particle migration and no sedimentation are observed, i.e., the material remains homogeneous in both the vertical and the radial directions, and thus the volume fractions are the same in both cases, i.e., $60 \%$. The system studied here is $40 \mu \mathrm{m}$ polystyrene beads suspended in a mixture of water and NaI; the latter allows to tune the density difference.

applied stress and thus the critical shear rate is very well defined experimentally as the lowest shear rate for which one can reach a steady state [Møller et al. (2006); Møller et al. (2008); Fall et al. (2009)]— see Fig. 2(a). We also showed that it is only at a homogeneous volume fraction that corresponds to random close packing that a yield stress emerges [Fall et al. (2009)]. Thus, we concluded that there are no particle contacts for the homogeneous system for volume fractions below random close packing, in line with its Newtonian rheology. By contact, we mean that particles interact continuously through lubricated or direct solid frictional/collisional contacts and percolating networks of particles form, evolve, and break continuously throughout the suspension [Ancey et al. (1999)].

For constant applied global shear rate, the coexistence of the flowing and nonflowing regime can be described in terms of a lever rule, in which the local shear rate inside the

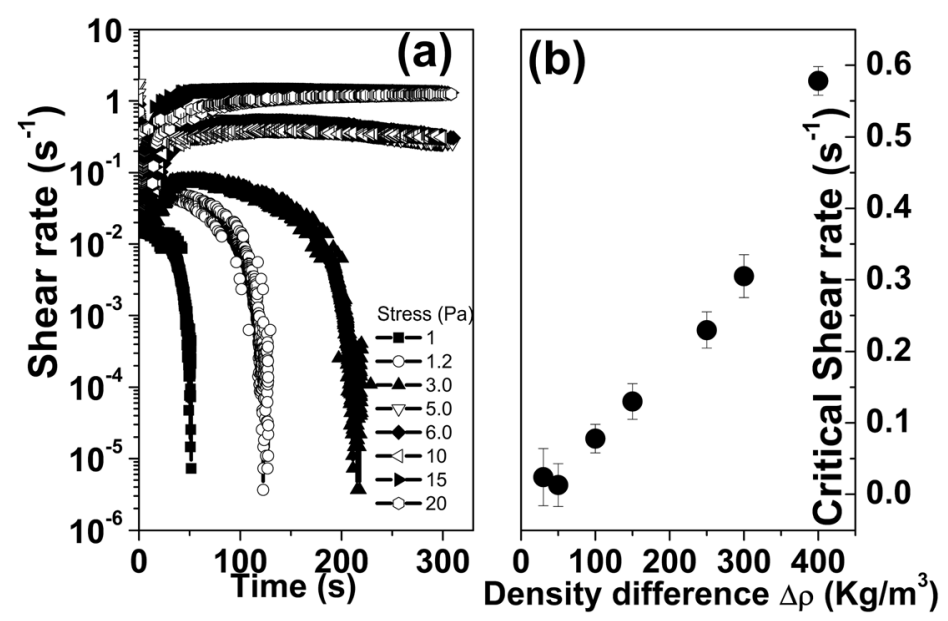

FIG. 2. (a) Shear rate vs time for different applied shear stresses in a mismatched suspension $\left(\Delta \rho=150 \mathrm{~kg} / \mathrm{m}^{3}\right)$. (b) Critical shear rate as a function of the density difference [Fall et al. (2009)]. 
high-shear band remains constant, as shown by systematic measurements on colloidal gels: Rheological measurements at a controlled strain rate show a stress plateau in the shear-banding regime. When the stress is imposed, the strain rate is not stable and evolves either toward a lower or an upper bound. The curve that separates these two cases exhibits a negative slope, associated with mechanical instabilities. The flow instabilities are absent above a critical shear rate, at which the flow curve recovers a positive slope [Møller et al. (2008)].

Moreover, as was shown in Fall et al. (2009), the viscosity bifurcation and the local measurements allow one to define a critical shear rate, as the lowest shear rate for which a steady state flow can be sustained; this critical rate is found to vary linearly with density difference [Fig. 2(b)]. This suggests that this critical strain rate is governed by a simple stress balance, where the viscous stresses $\approx \eta \dot{\gamma}$ are balanced by a compressive stress due to gravity. From dimensional analysis it follows that $\eta \dot{\gamma} \propto \Delta \rho g L$, with $L$ a length scale. What is this length scale $L$ ? Fall et al. (2009) suggested to take the particle radius $R$ for this, leading to $\eta \dot{\gamma}_{c}=\Delta \rho g R$. This was observed to give a reasonable estimate of the critical shear rate of Fig. 2(b). However, it is unclear why the particle radius and not the suspension height should enter this stress balance.

To probe the rheology of non-density-matched suspensions in more detail, and to probe the pressure scale that sets the critical flow rate, we performed a third series of experiments in a Couette geometry, this time varying the filling height of different non-density-matched granular suspensions. The granular suspension consists of $4.6 \mathrm{~mm}$ Polymethyl Methacrylate (PMMA) beads immersed in Triton X-100 (viscosity $\eta=0.23 \mathrm{~Pa} \mathrm{~s}, \Delta \rho=107 \mathrm{~kg} / \mathrm{m}^{3}$ at $25^{\circ} \mathrm{C}$ ), a similar granular suspension as the one studied in Dijksman et al. (2010). The rheological measurements are performed in a "home designed" geometry: A split-bottom Couette shear cell. The suspension is stored in an open-topped cubic box (width $150 \mathrm{~mm}$ and $120 \mathrm{~mm}$ height). A toothed metallic cylinder (radius $R_{s}=25 \mathrm{~mm}$, tooth dimension $=2.5 \mathrm{~mm}$ ) connected to a rheometer (Anton Paar MCR501) is used to shear the suspension at a constant rotation rate $\Omega$ (from $\Omega=10^{-4}$ to $1 \mathrm{rps})$. The filling height of the material is determined by weighing the particles $\left(H / R_{s}\right.$ is varied from 0.5 to 2 with an absolute error of $d / R_{s}=0.2$ ). The split-bottom Couette cell is then filled by adding a known weight of particles. The system is manually stirred, the top is flattened, and the filling height is re-measured with a ruler. The estimated initial volume fraction is $\varphi \approx 0.6$. For all the experiments, there is at least a $1 \mathrm{~cm}$ fluid layer over the top of the granular suspension. Experiments consists of a logarithmic ramping of the rotation speed $\left(3 \times 10^{-4}\right.$ to $0.3 \mathrm{rps}$ in 30 steps $)$ along with the time interval each speed is held. This time $\left(10^{3}-1 \mathrm{~s}\right)$ is chosen so that each rotation rate is held for 0.3 rotation. We do not observe significant effects in the results if the duration of the time intervals is made longer. In addition, experiments where the velocity is reversed (from 0.3 to $3 \times 10^{-4}$ ) present small hysteresis only. The system is thus in a steady-state at each rotation rate, and has the time to dilate at each step.

Rheological measurements are presented on Fig. 3(a), for various filling heights. We used a Herschel-Bulkley type model to fit the data

$$
T=T_{0}+K \Omega
$$

As reported earlier in Dijksman et al. (2010) with a similar suspension sheared in the splitbottom geometry, if we fit Eq. (2) to the data we find $b \approx 1$ for all $H / R_{s}$, i.e., a Bingham type of rheological law is measured, consistent with the data presented in Fig. 1.

A critical rotation rate can now be defined as the value of $\Omega$ where the first and second term in Eq. (2) balance, i.e., $\Omega_{c}=T_{0} / K$. As shown in Fig. 3(b), this critical strain rate 

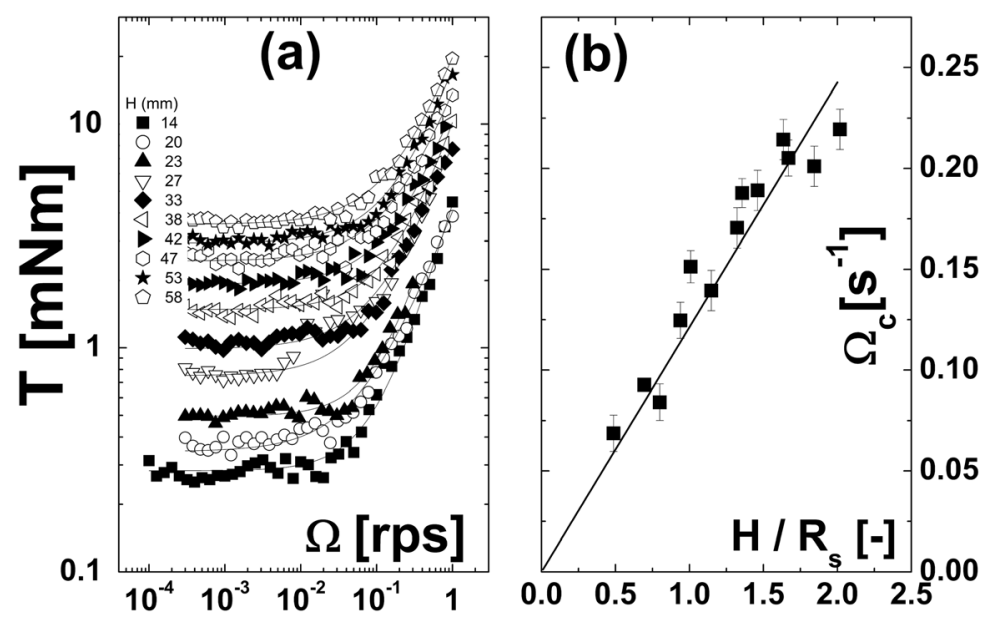

FIG. 3. Filling height dependence of the rheological measurements obtained in a Couette geometry with $4.6 \mathrm{~mm}$ diameter PMMA beads. (a) Torque as a function of the rotation rate of the Couette cylinder. Different symbols (see the legend) correspond to different filling heights. Lines are fits to a Bingham model of the form $T=T_{0}+K \Omega$. (b) Variation of the critical rotation rate $\Omega_{c}$ as a function of filling height-here $\Omega_{c}=T_{0} / K$. The solid line is a linear fit of the form $\Omega_{c}=(0.12 \pm 0.02) H / R_{s}$.

varies linearly with the filling height, ruling out that the characteristic length scale $L$ is the particle diameter.

\section{A SIMPLE SCALING MODEL}

The correct argument to obtain the critical shear rate is to focus on the balance of the static and dynamical stresses. Assuming an effective frictional picture to describe the rheological data, one can write [Dijksman et al. (2010), GDR Midi (2004), Unger et al. (2004)]

$$
\sigma=\mu(I) P \approx\left[\mu_{0}+\mu_{1} I\right] P
$$

where $\mu_{0}$ and $\mu_{1}$ are the static and dynamic friction coefficients (presumably having a similar value), respectively; and $I$ is a dimensionless number capturing the effective friction in suspension rheology [Cassar et al. (2005)]. The local static stress then equals $\mu_{0} P$, and the dynamic stress $\mu_{1} I P$ - using $I=\frac{\eta \dot{\gamma}}{\alpha P}$, where $\alpha$ is a permeability parameter, we find that the dynamic stress is of order $\mu_{1} \eta \dot{\gamma} / \alpha$ [Cassar et al. (2005)].

To set up a proper balance of the total stresses, we need to integrate the static and dynamic stresses over the depth of the suspension bed, and use that the relevant pressure $P$ is the granular pressure due to gravity, $P=\Delta \rho g(H-z)$ suppose that the concentration profile is homogeneous so that $\Delta \rho$ is constant [Fall et al. (2009)]. Equating the total static and dynamic stresses then yields

$$
\eta \dot{\gamma}_{c}=\Delta \rho g H\left(\frac{\alpha \mu_{0}}{2 \mu_{1}}\right)
$$

This can be compared, first, to the experimental data from the MRI rheology. The ratio $\mu_{0} / \mu_{1}$ is of order unity, allowing us to extract the value $\alpha \approx 0.0013$ from a linear fit to the data shown in Fig. 2(b). This estimate for $\alpha$ is in good agreement with the Carman-Kozeny equation [Carman (1956)], an extension of Darcy's law for flow through 
a porous medium that yields an explicit prediction for the proportionality constant between pressure drop and flow rate in Darcy's law

$$
\nabla p=\frac{45 U \eta}{R^{2}} \frac{(1-\varepsilon)^{2}}{\varepsilon^{3}}
$$

where $\nabla p$ is the pressure gradient, $U$ the average fluid velocity, and $\varepsilon$ the porosity of the bed. In our case, the MRI results indicate that when the packing is dense, the porosity is very close to that for random close packing of spheres, so we will use $\varepsilon=0.36$. Doing so, one obtains $\alpha \approx 0.00126$, in excellent agreement with our fitted value of $\alpha$.

We can also compare the individual fitting constants $T_{0}$ and $K$ that arise in the Bingham fit to the rheological data of Fig. 3(a) with the theoretical predictions. For our Couette cell with inner radius $R_{s}$, outer radius $R_{0}$, and suspension filling height $\mathrm{H}$, the torque is related to the stresses at the inner boundary as $T=2 \pi R_{s}^{2} \int_{0}^{H} d z \sigma(z)$.

Substituting Eq. (4), using $\mu_{1} I P=\frac{\mu_{1} \eta \dot{\gamma} P}{\alpha P}=\frac{\mu_{1} \eta \dot{\gamma}}{\alpha}$, using the equations for the hydrostatic pressure, and assuming that the shear rate is constant throughout the gap and given as $\dot{\gamma}=\Omega R_{S} /\left(R_{0}-R_{S}\right)$ we obtain $T=2 \pi R_{s}^{2}\left[\int_{0}^{H}\left[\mu_{0} \Delta \rho g(H-z)+\frac{\mu_{1} \eta}{\alpha} \dot{\gamma}\right] d z\right]$. Performing the integral yields:

$$
T=\mu_{0} \pi \Delta \rho g R_{s}^{2} H^{2}+2 \pi \frac{\mu_{1} \eta}{\alpha} R_{s}^{2} H \frac{R_{s}}{R_{0}-R_{s}} \Omega .
$$

Identifying this expression with the Bingham fit, $T=T_{0}+K \Omega$, we readily obtain

$$
T_{0}=\mu_{0} \Delta \rho g \pi R_{s}^{4}\left(\frac{H}{R_{s}}\right)^{2}
$$

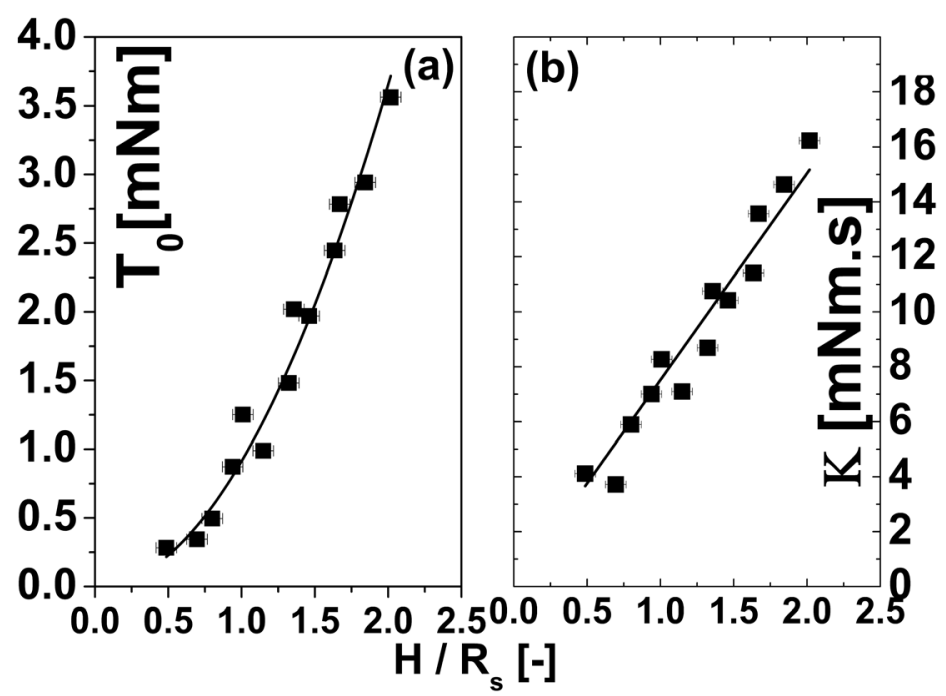

FIG. 4. (a) Height dependence of the yield torque $T_{0}$. The line is a parabolic fit of the data of the form, as expected from Eq. (7). In good approximation, $T_{0}=\left(0.9 \pm 0.1 \times 10^{-3}\right) \mathrm{Nm}\left(H / R_{s}\right)^{2}$. (b) Effective viscosity of the suspension as a function of the filling height. The solid line is a linear fit of the data of the form $K=\left(7.5 \pm 1 \times 10^{-3}\right) \mathrm{Nm} \mathrm{s}\left(H / R_{S}\right)$ as expected from Eq. (8). 


$$
K=\mu_{1} \frac{\eta}{\alpha} 2 \pi R_{s}^{3}\left(\frac{H}{R_{S}}\right)\left(\frac{R_{s}}{R_{0}-R_{S}}\right) .
$$

This can be directly compared to the data. Figure 4(a) indeed shows a quadratic increase of $T_{0}$ with $\left(H / R_{S}\right)$, and substituting our experimental values in Eq. (7), we find that $T_{0} \approx 10^{-3} \mu_{0}\left(H / R_{s}\right)^{2}$ which is consistent with our fit shown in Fig. 4(a), provided that $\mu_{0}$ is of order $1\left(\mu_{0}=0.7 \pm 0.1\right)$ : This value is in agreement with the measured static friction coefficient of PMMA [Archard (1957)]. Similarly, we indeed observe a linear dependence of the "effective viscosity" $K$ with the filling height $H$ in Fig. 4(b), as predicted by Eq. (8), and substituting our experimental values in Eq. (8), we find that $K \approx 8.5 \times 10^{-3}$ $\mu_{1}\left(H / R_{s}\right)$, which is consistent with the fit shown in Fig. 4(b), provided that $\mu_{1}$ is of order 1. Finally, we can define a critical rotational rate $\Omega_{c}$ as the rotation rate for which "frictional" and "viscous" regime overlap, i.e., $T_{0}=K \Omega_{c}$, and taking $\mu_{0}=\mu_{1}$, it follows that

$$
\Omega_{c}=\frac{\mu_{0}}{\mu_{1}} \frac{\alpha \Delta \rho g H}{2 \eta} \approx \dot{\gamma}_{c} .
$$

If we do so with a density difference of $110 \mathrm{~kg} / \mathrm{m}^{3}$, we obtain [from Fig. 2(b)] $\dot{\gamma}_{c}=0.65 \Omega_{c}$, in very reasonable agreement with the experimental observations. Thus, this simple scaling argument seems to be sufficient to account for all of the experimental observations presented in this paper.

\section{CONCLUSION}

We have compared different experiments, using different techniques and performing the measurements on different systems to study the behavior of non-density-matched non-Brownian suspensions. The absence of Brownian motion, together with the buoyancy and gravitational forces working on the particles, makes that large gradients in particle concentration develop in the direction parallel to gravity. The particles sediment or cream, which leads to a part of the system that is so dense in particles that it develops a yield stress. On the other hand, if the flows are sufficiently rapid, they homogenize the system. This naturally leads to the viscosity bifurcation, which in turn leads to the appearance of a critical shear rate above which the suspension is homogenized by the flow, and below which sedimentation or creaming happens. What exactly fixes the value of this critical shear rate was an outstanding question in the literature. Our experiments show that the value of the critical shear rate and its dependence on the experimental parameters are governed by a competition between the viscous and the gravitational forces. This allows us to present a simple scaling model that predicts the value of the critical shear rate, as a function of the experimental parameters. It is found that this approach suffices to account for the experimental results from different types of experiments (local and global) in different setups and systems.

\section{ACKNOWLEDGMENTS}

The authors thank William Derek Updegraff from U. Maryland who performed the Triton Couette experiments. This work is part of the NWO-FOM program "Jamming and Rheology." 


\section{References}

Ancey, C., and P. Coussot, "Transition frictionnelle/visqueuse pour une suspension granulaire," C. R. Acad. Sci., Sér. IIb Mec. Phys. Astron. 327, 515-522 (1999).

Ancey, C., P. Coussot, and P. Evesque, "A theoretical framework for very concentrated granular suspensions in a steady simple shear flow,” J. Rheol. 43, 1673-1699 (1999).

Archard, J. F., "Elastic deformation and the laws of friction," Proc. R. Soc. London 243(1233), 190-205 (1957).

Barentin, C., E. Azanza, and B. Pouligny, "Flow and segregation in sheared granular slurries," Europhys. Lett. 66, 139-145 (2004).

Bonn, D., S. Rodts, M. Groenink, S. Rafai, N. S. Bonn, and P. Coussot, "Some applications of magnetic resonance imaging in fluid mechanics: Complex flows and complex fluids," Annu. Rev. Fluid Mech. 40, 209-233 (2008).

Bonnoit, C., J. Lanuza, A. Lindner, and E. Clement, "Mesoscopic length scale controls the rheology of dense suspensions," Phys. Rev. Lett. 105, 108302 (2010a).

Bonnoit, C., T. Darnige, E. Clement, and A. Lindner, "Inclined plane rheometry of a dense granular suspension," J. Rheol. 54, 65-79 (2010b).

Boyer, F., É. Guazzelli, and O. Pouliquen, “Unifying suspension and granular rheology,” Phys. Rev. Lett. 107, 188301 (2011).

Brown, E., and H. M. Jaeger, "Dynamic jamming point for shear thickening suspensions," Phys. Rev. Lett. 103, 086001 (2009).

Brown, E., and H. M. Jaeger, "Through thick and thin,” Science 333, 1230-1231 (2011).

Brown, E., and H. M. Jaeger, "The role of dilation and confining stresses in shear thickening of dense suspensions," J. Rheol. 56, 875-923 (2012).

Brown, E., N. A. Forman, C. S. Orellana, H. Zhang, B. W. Maynor, D. E. Betts, J. M. DeSimone, and H. M. Jaeger, "Generality of shear thickening in dense suspensions," Nature Mater. 9, 220-224 (2010).

Carman, P. C., Flow of Gases Through Porous Media (Butterworths, London, 1956), Chap. 1.

Cassar, C., Nicolas, M., and Pouliquen, O., "Submarine granular flows down inclined planes," Phys. Fluids 17, 103301 (2005).

Cheng, X., J. H. McCoy, J. N. Israelachvili, and I. Cohen, "Imaging the microscopic structure of shear thinning and thickening colloidal suspensions," Science 333, 1276-1279 (2011).

Dijksman, J. A., E. Wandersman, S. Slotterback, C. R. Berardi, W. D. Updegraff, M. van Hecke, and W. Losert, "From frictional to viscous behavior: Three-dimensional imaging and rheology of gravitational suspensions," Phys. Rev. E 82, 060301(R) (2010).

Dijksman, J. A., G. H. Wortel, L. T. H. van Dellen, O. Dauchot, and M. van Hecke, "Jamming, yielding and rheology of weakly vibrated granular media," Phys. Rev. Lett. 107, 108303 (2011).

Fall, A., F. Bertrand, G. Ovarlez, and D. Bonn, "Yield stress and shear banding in granular suspensions," Phys. Rev. Lett. 103, 178301 (2009).

Fall, A., F. Bertrand, G. Ovarlez, and D. Bonn, "Shear thickening and migration in granular suspensions," Phys. Rev. Lett. 105, 268303 (2010).

Fall, A., F. Bertrand, G. Ovarlez, and D. Bonn, "Shear thickening in cornstarch suspension,” J. Rheol. 56(3), 575-591 (2012).

Fall, A., N. Huang, F. Bertrand, G. Ovarlez, and D. Bonn, "Shear thickening of cornstarch suspensions as a reentrant jamming transition," Phys. Rev. Lett. 100, 018301 (2008).

Fenistein, D., J.-W. van de Meent, and M. van Hecke, "Universal and wide shear zones in granular bulk flow," Phys. Rev. Lett. 92, 094301 (2004).

Fenistein, D., J.-W. van de Meent, and M. van Hecke, "Core precession and global modes in granular bulk flow,” Phys. Rev. Lett. 96, 118001 (2006).

Fenistein, D., and M. van Hecke, "Wide shear zones in granular bulk flow," Nature (London) 425, 256 (2003).

GDR, MiDi, “On dense granular flows,” Eur. Phys. J. E 14, 341-365 (2004).

Goldhirsch, I., "Rapid granular flows," Annu. Rev. Fluid Mech. 35, 267-293 (2003).

Huang, N., and D. Bonn, "Viscosity of a dense suspension in Couette flow," J. Fluid Mech. 590, 497-507 (2007). 
Huang, N., G. Ovarlez, F. Bertrand, S. Rodts, P. Coussot, and D. Bonn, "Flow of wet granular materials," Phys. Rev. Lett. 94, 028301 (2005).

Husband, D. M., N. Aksel, and W. Gleissle, "The existence of static yield stresses in suspensions containing noncolloidal particles,” J. Rheol. 37, 215-235 (1993).

Jaeger, H., S. Nagel, and B. Behringer, "Granular solids, liquids, and gases," Rev. Mod. Phys. 68, 1259-1273 (1996).

Jop, P., Y. Forterre, and O. Pouliquen, “A constitutive law for dense granular flows," Nature 441, 727-730 (2006).

Møller, P. C. F., A. Fall, V. Chikkadi, D. Derks, and D. Bonn, “An attempt to categorize yield stress fluid behaviour,” Philos. Trans. R. Soc. London, Ser. A 367, 5139-5155 (2009).

Møller, P. C. F., J. Mewis, and D. Bonn, "Yield stress and thixotropy: On the difficulty of measuring yield stresses in practice," Soft Matter 2, 274-283 (2006).

Møller, P. C. F., S. Rodts, M. A. J. Michels, and D. Bonn, "Shear banding and yield stress in soft glassy materials," Phys. Rev. E 77, 041507 (2008).

Morris, J. F., and F. Boulay, "Curvilinear flows of noncolloidal suspensions: The role of normal stresses," J. Rheol. 43, 1213-1237 (1999).

Ovarlez, G., F. Bertrand, and S. Rodts, "Local determination of the constitutive law of a dense suspension of noncolloidal particles through magnetic resonance imaging," J. Rheol. 50, 259-292 (2006).

Prasad, D., and H.-K. Kytömaa, "Particle stress and viscous compaction during shear of dense suspensions," Int. J. Multiphase Flow 21, 775-785 (1995).

Rodts, S., F. Bertrand, S. Jarny, P. Poullain, and P. Moucheront, "Développements récents dans l'application de l'IRM a la rhéologie et a la mécanique des fluides," C. R. Chim. 7, 275-282 (2004).

Schall, P., and M. van Hecke, "Shear bands in matter with granularity," Annu. Rev. Fluid Mech. 42, 67-88 (2010).

Unger, T., J. Torök, J. Kertësz, and D. E. Wolf, "Shear band formation in granular media as a variational problem,” Phys. Rev. Lett. 92, 214301 (2004).

Zarraga, I. E., D. A. Hill, and D. T. Leighton, "The characterization of the total stress of concentrated suspensions of noncolloidal spheres in Newtonian fluids,” J. Rheol. 44, 185-220 (2000). 